\title{
Prolonged head down bed rest-induced inactivity impairs tonic autonomic regulation while sparing oscillatory cardiovascular rhythms in healthy humans
}

\author{
Guido Ferretti $^{\mathrm{a}, \mathrm{b}}$, Ferdinando lellamo ${ }^{\mathrm{c}, \mathrm{d}}$, Paolo Pizzinelli ${ }^{\mathrm{e}}$, Marcel A. Kenfack ${ }^{\mathrm{a}}$, \\ Frédéric Lador ${ }^{\mathrm{a}}$, Daniela Lucini ${ }^{\mathrm{f}}$, Alberto Porta ${ }^{\mathrm{g}}, \mathrm{Krzysztof} \mathrm{Narkiewicz}^{\mathrm{h}}$ and \\ Massimo Pagani ${ }^{\mathrm{e}, \mathrm{f}}$
}

Background Physical inactivity represents a major risk for cardiovascular disorders, such as hypertension, myocardial infarction or sudden death; however, underlying mechanisms are not clearly elucidated. Clinical and epidemiological investigations suggest, beyond molecular changes, the possibility of an induced impairment in autonomic cardiovascular regulation. However, this hypothesis has not been tested directly.

Methods Accordingly, we planned a study with noninvasive, minimally intrusive, techniques on healthy volunteers.

Participants were maintained for 90 days strictly in bed, $24 \mathrm{~h}$ a day, in head-down $\left(-6^{\circ}\right)$ position (HDBR). Physical activity was thus virtually abolished for the entire period of HDBR. We examined efferent muscle sympathetic nerve activity, as a measure of vascular sympathetic control, baroreceptor reflex sensitivity, heart rate variability (assessing cardiovagal regulation), RR and systolic arterial pressure and low-frequency and high-frequency normalized components (as a window on central oscillatory regulation). Measures were obtained at rest and during simple maneuvers (moderate handgrip, lower body negative pressure and active standing) to assess potential changes in autonomic cardiovascular responsiveness to standard stimuli and the related oscillatory profiles.

Results HDBR transiently reduced muscle sympathetic nerve activity, RR, heart rate variability and baroreceptor reflex sensitivity late during HDBR or early during the recovery phase. Conversely, oscillatory profiles of RR and systolic arterial pressure variability were maintained throughout. Responsiveness to test stimuli was also largely maintained.

\section{Introduction}

Physical inactivity is a recognized risk factor for cardiovascular diseases and adverse cardiovascular events [1,2]. Conversely, physical exercise is not only associated to longer life expectancy [3], but also to a number of health benefits [4], among which improvements in neural cardiovascular regulation play a relevant role, as indicated by several studies showing that exercise training leads to a reduction in arterial pressure [5], an increase in baroreflex gain [5] and in heart rate variability [6], and a reduction in resting heart rate [6], an independent risk factor in cardiovascular disease [7].
Conclusion Prolonged inactivity as induced by HDBR in healthy volunteers reduces both cardiovagal and vascular sympathetic regulation, while largely maintaining peripheral responsiveness to standardized stimuli and sparing the functional structure of central oscillatory cardiovascular regulation. J Hypertens 27:551-561 @ 2009 Wolters Kluwer Health | Lippincott Williams \& Wilkins.

Journal of Hypertension 2009, 27:551-561

Keywords: autonomic nervous system, baroreflex, cardiovascular oscillations, cardiovascular regulation, inactivity, sympathetic regulation, vagal regulation

Abbreviations: ANS, autonomic nervous system; ASI, Agenzia Spaziale Italiana; BDC, baseline data collection; BRS, baroreceptor reflex sensitivity; DCMC, disorders of motor and cardiorespiratory control; HDBR, head down bed rest; HRV, heart rate variability; ICC, intraclass correlation coefficients; $K^{2}$, squared coherence; LBNP, lower body negative pressure; LCD, liquid crystal display; MSNA, muscle sympathetic nerve activity; REC, recovery; RESP, respiratory rate; SAP, systolic arterial pressure; SD, standard deviation; SE, standard error

${ }^{a}$ Département de Neurosciences Fondamentales, Centre Médical Universitaire,

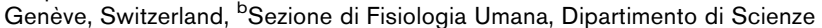
Biomediche e Biotecnologie, Università di Brescia, Brescia, 'Dipartimento di Medicina Interna, Università di Roma Tor Vergata, d IRCCS San Raffaele Pisana, Rome, ${ }^{e} \cup O$ Telemedicina, Ospedale L Sacco, Polo Universitario, ${ }^{f}$ Centro di Ricerca sulla Terapia Neurovegetativa, Dipartimento Scienze Cliniche, Università di Milano, ${ }^{9}$ Dipartimento di Tecnologie applicate alla salute, Università di Milano, Milan, Italy and hypertension Unit, Department of Hypertension and Diabetology, Medical University of Gdansk, Gdansk, Poland

Correspondence to Professor Massimo Pagani, Centro di Terapia Neurovegetativa, Università di Milano, Ospedale L. Sacco, Via G.B. Grassi 74 I-20157 Milano, Italy

Tel: +3902 39042802; e-mail: mp@ctnv.unimi.it

Received 14 March 2008 Revised 7 November 2008

Accepted 14 November 2008

Compared with the number of studies addressing the beneficial effects of regular physical activity, there are relatively few studies addressing directly the effects of prolonged inactivity on autonomic cardiovascular regulation, probably because of the technical difficulties entailed by maintaining strict inactivity in humans. In this context, studies on simulated microgravity, as with head down bed rest (HDBR) at $-6^{\circ}$, might offer a useful experimental model of inactivity [8]. In fact, with HDBR, subjects are kept strictly in bed for $24 \mathrm{~h}$ a day, for periods up to several months, thus inducing over time a marked 
impairment of muscular force and mass [9], together with a reduced exercise capacity [10]. However, as more recently summarized by Pavy-Le Traon et al. [11], this model of microgravity combines inactivity with a host of other effects on the circulation, and fluid and electrolyte balance that result from postural changes, and are mediated by gravity pull on the blood column. It should be mentioned that these effects (early central fluid shift followed, in time, by a decrease in total blood volume [9] with its possible influences on cardiac volume receptors) are modulated according to the specific position (head-up tilt, horizontal or head-down tilt).

Several studies addressed the changes produced by exposure to HDBR on autonomic nervous system (ANS) regulation, because of their reversibility and of the recognized importance of initial ANS changes in several conditions, such as arterial hypertension. In clinical studies, ANS cardiovascular regulation may be gleaned by analysis of the variability of continuous series of RR interval and systolic arterial pressure (SAP) over several minutes of length, employing mathematical models of various complexities, either in time or in frequency domain, or both. Time domain methods provide measures of average levels (tonic) (mean and SD), whereas frequency domain methods provide quantitative measures of oscillations (in either absolute or normalized units) around the mean.

HDBR has been consistently reported to induce a reduction of the time domain measure of baroreflex gain [12], implying an impairment of tonic vagal cardiac regulation. An accompanying impairment of sympathetic vascular regulation after inactivity would be suggested by a reduced pressor responsiveness to simple excitatory stimuli, such as handgrip $[13,14]$ and by a reduced norepinephrine turnover [15] following HDBR.

Surprisingly, direct studies on average muscle sympathetic nerve activity (MSNA) provide conflicting results, as an increase [16], a reduction [7] or no change [13] in MSNA has been reported after HDBR. Data regarding the oscillatory properties of neural cardiac regulation, as assessed indirectly by spectral analysis of RR variability, are also conflicting, as the low-frequency component has been described as increased [17] or unchanged [14] after HDBR. Virtually no data are available on oscillatory properties of MSNA after HDBR.

The recent observations that in a variety of cardiovascular diseases, such as cardiac transplantation [18], childhood hypertension [19] and the metabolic syndrome [20], indices of average vagal or sympathetic activity might be altered whereas oscillatory indices are not, on one hand furthers the concept that these two different modalities of neural cardiovascular regulation might code different functions [21], and on the other leaves open the possibility that HDBR also might differently affect tonic and oscillatory autonomic cardiovascular regulation, possibly by way of an induced insulin resistance [22].

Accordingly, we planned the present investigation, employing noninvasive, minimally intrusive, multiparameter techniques, on healthy volunteers, in order to minimize the confounding effects of altered base line autonomic balance as it would occur with patients. Our aim was to assess whether prolonged HDBR, with its inherent inactivity, would produce a selective impairment of indices of tonic sympathetic vascular and cardiac vagal autonomic regulation, while largely maintaining the functional dynamics of central oscillatory cardiovascular regulation and peripheral responsiveness to standardized excitatory stimuli.

\section{Methods}

\section{Participants}

Seventeen healthy men participated in this study. They were $31.9 \pm 4$ years old, $174.5 \pm 4.1 \mathrm{~cm}$ tall and weighed $71.3 \pm 5 \mathrm{~kg}$. None of the participants was taking cardiovascular medication at the time of the study, and all were nonsmokers. During the selection procedure, each volunteer was interviewed and had a general physical examination that included blood and urine chemistries. At least $24 \mathrm{~h}$ elapsed from any exercise foreseen for other experiments and our recordings.

The bed-rest investigation was conducted with the facilities of the Space Clinic, Institute of Space Medicine and Physiology (MEDES), Toulouse, France, as part of a series of European Space Agency (ESA)-sponsored investigations on the effects of simulated microgravity. At the screening procedure, volunteers were informed of all procedures and risks associated with the experimental testing. Informed consent was obtained from each volunteer. The study was conducted in accordance with the Declaration of Helsinki, and ethical committee approval was previously obtained.

\section{Protocol}

The bed-rest program design consisted of 120 days' period of continued stay in the MEDES facilities: a 15-day run in control period, followed by a 90 -day period of bed rest in the $-6^{\circ}$ head-down tilt position (HDBR), followed by an additional 15 days of early recovery (REC) evaluation. During the 15 days run-in and during the REC periods, volunteers were allowed to freely perform daily life activities but were asked to avoid regular, scheduled physical exercise. Long-term REC evaluations were effected until 360 days of post-bed-rest REC with programmed visits. During HDBR, volunteers were kept all time in bed, under close television surveillance, and were never allowed to get out. An echo Doppler examination, to exclude venous anomalies at the time of enrolment, in addition to daily examination by the 
in-charge physician, coupled with calf massage, passive mobilization of joints and diaphragmatic breathing sessions, was programmed in order to minimize deep vein thrombosis risk.

Resting measurements were performed 1 day before HDBR, on day 89 during HDBR and on days 5 and 30 during REC after HDBR $(\mathrm{REC}+5$ and REC +30 , respectively). Responses to standardized stimuli, such as static handgrip, active standing and lower body negative pressure (LBNP) were performed 1 day before HDBR and after HDBR at REC +5 and $\mathrm{REC}+30$. Due to overall plan of the bed-rest study which included several experiments, half of the participants were studied between 09.00 and $11.00 \mathrm{~h}$ and half between 15.00 and $17.00 \mathrm{~h}$. Each participant carried out the protocol always at the same time of day. All experiments were carried out in a thermostated room, where the temperature was fixed at $24^{\circ} \mathrm{C}$.

\section{Resting measurements}

After instrumentation and after $5 \mathrm{~min}$ of habituation at quiet rest, recordings were obtained during $5 \mathrm{~min}$ of quiet rest.

\section{Handgrip experiment}

After instrumentation and after $5 \mathrm{~min}$ of habituation at quiet rest, recordings were obtained during $5 \mathrm{~min}$ of supine rest, $5 \mathrm{~min}$ of handgrip contraction maintained always at $25 \%$ of the individual maximal voluntary contraction and during $5 \mathrm{~min}$ of REC. Handgrip was performed by means of a portable dynamometer, which was previously tested for linearity. Participants maintained the target force during handgrip contraction by aid of a liquid crystal display (LCD) visual feedback placed in front of them. The target force was such that all participants could maintain the same stimulus for 5 min, independent of physical condition, before (control period) and after bed rest.

\section{Active standing experiment}

For this part of the protocol, volunteers were asked to stand up unaided after $10 \mathrm{~min}$ of supine posture and to maintain the upright posture for $5 \mathrm{~min}$, during which data were recorded.

\section{Lower body negative pressure experiment}

Participants entered a steel cylindrical device that was sealed to the iliac crest and was connected to a negative pressure pump, which generated successive ramps of greater negativity up to $-60 \mathrm{mmHg}$. This value was the maximum allowed by the ethics committee. Each step was maintained for $5 \mathrm{~min}$ and was followed by a 5 -min rest at $0 \mathrm{mmHg}$. Only data obtained at the minimum negative pressure (i.e., $-60 \mathrm{mmHg}$ ) are presented in this study, for simplicity.

\section{Recorded variables}

During the experiments, the ECG trace from a precordial lead, respiratory rate (RESP) by a thoracic belt (Biopac Systems, Goleta, California, USA) and indirect noninvasive finger blood pressure (Finapres, Ohmeda 2350, Englewood, Colorado, USA) were continuously recorded. In addition, before and after the bed-rest period, continuous recordings of MSNA were obtained from the peroneal nerve. A tungsten microelectrode with a shaft diameter of $200 \mu \mathrm{m}$, tapering to an uninsulated tip of $1-5 \mu \mathrm{m}$, was inserted percutaneously into the muscle nerve fascicles of the peroneal nerve without anesthesia. A subcutaneous reference electrode was inserted $2-3 \mathrm{~cm}$ away from the recording electrode. After preamplification, sympathetic bursts were identified by inspection of the mean voltage neurogram. All recorded signals were digitalized (MP150; Biopac Systems) at a sampling rate of $300 \mathrm{~Hz}$, fed to a computer and stored for off-line analysis.

\section{Data treatment}

A purposely developed software (Heartscope, ver.1.6, A.M.P.S. llc, New York, USA) was used to identify the peak of R wave on ECG, the SAP, bursts of MSNA and RESP. The software constructs automatically time series of RR intervals, SAP, MSNA and RESP, with low operator-analysis interaction. Spontaneous variability of RR interval, SAP, MSNA and RESP was evaluated by means of power spectral analysis using an autoregressive algorithm on all recorded parameters, as previously described [23]. In short, from beat-to-beat variability series of adequate length and stationarity (usually 250350 beats), the software calculated simple statistics and the best autoregressive estimate of the power spectral density. The total power of RR and SAP variabilities, corresponding to variance, was initially obtained [23]. Subsequently, the powers and frequencies of the low $(0.03-0.14 \mathrm{~Hz})$ and the high $(0.15-0.5 \mathrm{~Hz})$ frequency spectral components (low frequency and high frequency, respectively), expressed in absolute and normalized units, were computed as the ratio of the absolute power of either high frequency or low frequency to the total power, subtracting the very-low-frequency component, and multiplying this ratio by 100 . To this purpose, we need to point out that, when using autoregressive algorithms capable to specifically detect power and center frequency of low-frequency and high-frequency components, the sum of low frequency and high frequency does not usually account for all spectral powers, because of the frequent presence of several small, nonsignificant, noise components [23]. The integrated baroreflex control of heart rate was assessed by means of the sequence method [24]. Briefly, the beat-by-beat time series of SAP and RR interval were searched for sequences of three or more consecutive beats in which SAP and RR interval changed in the same direction, either increasing or decreasing (i.e., $+\mathrm{RR} /+\mathrm{SAP}$ and $-\mathrm{RR} /-\mathrm{SAP}$ ). These sequences were identified as baroreflex sequences. A 
linear regression was applied to each individual sequence, and only those sequences in which the value of $r^{2}$ was more than 0.85 were accepted and the slope calculated. The mean individual slope of SAP/RR interval relationship, obtained by averaging all slopes computed within a given test period, was calculated and taken as a measure of the integrated spontaneous baroreceptor reflex sensitivity (BRS) for that period.

Traditional analysis of MSNA is based on visual computation of the rate (bursts/min) and amplitude of the bursts. In the present study, the software automatically detected bursts and computed amplitude and area of the bursts. A burst in neural activity was recognized when it exceeded a threshold in amplitude and duration (usually more than three-fold the noise), both continuously updated during the analysis. The average number of bursts in a time unit (minutes or 60 beats) was derived and expressed accordingly in bursts/min or bursts/ 60 beats. The average amplitude and area in each condition were normalized by the average amplitude and area in the resting condition to account for the known baseline variations. Thus, they were dimensionless. In addition, the mean MSNA values were calculated on a beat-to-beat basis as the defined integral of the MSNA signal over the current heart period divided by its duration, thus deriving a MSNA variability series synchronous with SAP and RR variability signals [25].

Cross-spectral analysis was used to determine whether there was a stable relationship between RR and SAP and MSNA series to broaden the information on regulated cardiovascular stability (Fig. 1). Cross-spectral analysis was performed by means of bivariate autoregressive identification and was used to compute a squared coherence function $\left(K^{2}\right) . K^{2}$ was defined as the squared crossspectrum amplitude normalized by the product of the spectra of two signals. $K^{2}$ is a measure of the statistical link between RR, SAP and MSNA series at any given frequency and ranges between 1 (perfect correlation) and 0 (perfect uncorrelation). In this study, coherence value of at least 0.5 was considered significant [26]. Lowfrequency $K^{2}$ and high-frequency $K^{2}$ values were derived as the maximum of $K^{2}$ inside the low-frequency and high-frequency bands, respectively.

\section{Statistics}

All values are reported as means $\pm \mathrm{SE}$. The significance of the differences in the reported variables at rest among the different experimental periods was assessed using a mixed model analysis complemented by Hotelling Test. However, for clarity, data are presented separately for pre-HDBR against post-HDBR and for pre-HDBR versus HDBR periods. Relationships between variables were analyzed with Pearson correlation; constancy of findings was assessed with intraclass correlation [27]. The level of statistical significance was set at a $P$ value of less than 0.05 (two sided). All statistical operations were performed with a commercial statistical package (SPSS, Inc., Chicago, Illinois, USA, version 13).

\section{Results}

Table 1 reports summary rest hemodynamic and autonomic data obtained at baseline (BDC), late $(+89$ days) during HDBR and early ( +5 days) into REC. It is evident that RR is reduced both during and after HDBR; at this latter time, MSNA also (either in burst/min or burst/ 60 beats) is reduced $(P<0.017$, both cases, Monte Carlo procedure). RR variance appears reduced only during HDBR, concomitant with a significant reduction of BRS, suggesting cardiac vagal withdrawal. Variables returned at pre-HDBR, BDC values when reassessed at +30 days of REC (data not shown). It is also worth noticing the high coherence value (Fig. 1 and Table 1) at low frequency and high frequency between spectral components for paired variables (RR and SAP, RR and MSNA, SAP and MSNA). In the case of MSNA, the coherence appears somewhat more elevated at low frequency.

\section{Effects of head down bed rest on responses to excitatory stimuli \\ Handgrip}

Handgrip (Table 1) maintained at $25 \%$ of maximal voluntary contraction provoked small expected changes in RR interval (from $930 \pm 34$ to $840 \pm 22 \mathrm{~ms}$ ) and in SAP (from $126 \pm 4$ to $138 \pm 5 \mathrm{mmHg}$ ) during the BDC period (Fig. 2). These changes appeared to only slightly reduce the $\mathrm{REC}+5$. Handgrip had small effects on average MSNA, as expected. MSNA was however clearly reduced (both in burst/min and burst/60 beats) in REC +5 and returned toward pre bed-rest value at $\mathrm{REC}+30$. The spectral profiles of RR or MSNA variability were not affected by handgrip (Fig. 3).

\section{Lower body negative pressure and active orthostatism}

Figure 3 depicts changes in RR and SAP values and their variability indices produced by $-60 \mathrm{mmHg}$ of LBNP, that is, the maximal-tolerated level, and, by active standing, before and after HDBR.

Notice the clear, and concordant, reductions in RR, BRS and the high-frequency component (normalized units) of RR. Conversely, RR low frequency (normalized units) and SAP low frequency $\left(\mathrm{mmHg}^{2}\right)$ appear concordantly increased. These changes were not different if examined before or after HDBR. It should also be noted that active standing produced larger changes as compared with LBNP $(-60 \mathrm{mmHg})$. SAP was instead essentially unmodified.

\section{Intraclass correlation coefficient analysis}

Figure 4 depicting intraclass correlation coefficients obtained from resting values throughout the experimental period strongly suggests that both average and oscillatory 


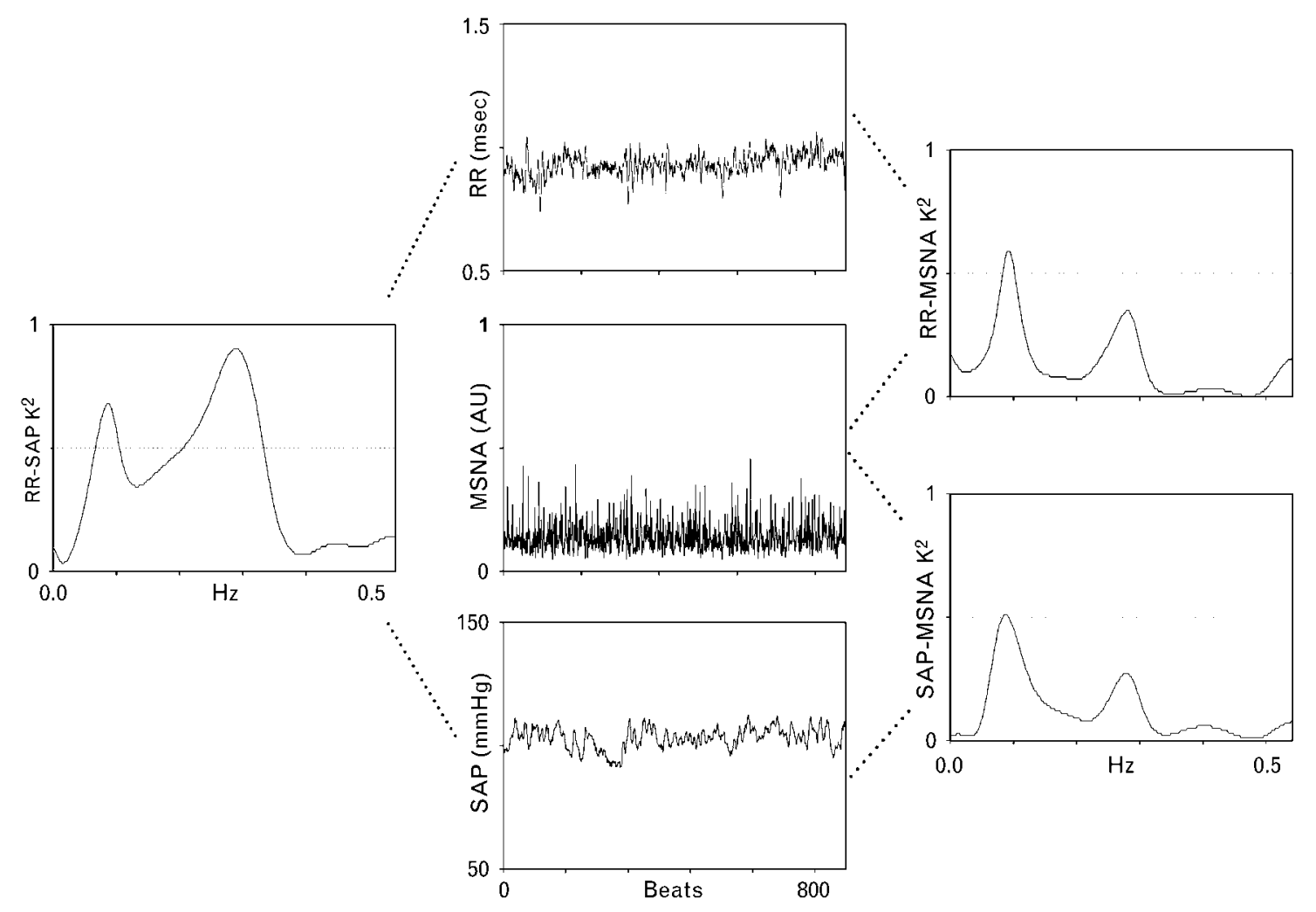

Example obtained from one participant of cross-spectra plots of the squared coherence between RR interval and systolic arterial pressure (left panel), between RR interval and muscle sympathetic nerve activity (right, top panel) and between systolic arterial pressure and muscle sympathetic nerve activity (right, bottom panel). RR interval, MSNA and SAP series are shown in the central panels (from top to bottom, respectively). The dotted lines indicate the threshold value (0.5) above which the correlation between oscillations is significant. Notice that for all pairs of data, there are two peaks in the squared coherence function, one at LF and one at HF. $K^{2}$, squared coherence; HF, high frequency; LF, low frequency; MSNA, muscle sympathetic nerve activity; RR; SAP, systolic arterial pressure.

variables maintain a consistent structure under these experimental conditions.

\section{Discussion}

The study reports the effect of prolonged $-6^{\circ} \mathrm{HDBR}$ and attendant inactivity both on cardiovascular variables and on muscle peripheral sympathetic efferent nerve activity (MSNA) along with their oscillatory rhythms both in resting conditions and in response to standardized excitatory stimuli. We show that the prolonged HDBR produce a clear reduction in directly recorded peripheral sympathetic nerve activity, RR variance and BRS in resting conditions, whereas oscillatory dynamics of central neural cardiovascular regulation appear maintained.

\section{Head down bed rest}

HDBR is frequently employed as a model of microgravity, combining the effects of inactivity with those of the release from gravitational pull on the blood column. The combination of these factors in prolonged HDBR leads to progressive reductions in muscular and cardiorespiratory performance, providing a convenient experimental model of reversible changes in regulatory mechanisms. Prolonged HDBR projects are technically unique because maintaining participants strictly in bed requires highly organized personnel and professional skills. Regarding HDBR-related cardiovascular studies, the analysis of cardiovascular variability signals has been used as a tool to investigate autonomic regulation $[25,28,29]$ with conflicting results $[14,17]$. With HDBR, a clear-cut reduction of $\mathrm{RR}$ variance and $\mathrm{BRS}$ was observed (Table 1), suggesting that this intervention impairs tonic cardiac vagal regulation, in line with most prior investigations [12,30]. These changes in the amplitude of time indices [31] were rapidly followed by REC.

The present investigation provides new insights into tonic sympathetic efferent activity. In fact, average values of MSNA appeared reduced 5 days into REC from HDBR, with a return to baseline after 30 days of $\mathrm{REC}$, in line with the results reported by Shoemaker $e t$ al. [32] after a much shorter period of HDBR. Our finding was obtained by measuring activity both in burst $/ \mathrm{min}$ as well as in burst/60 beats, which accounts for differences in 


\begin{tabular}{|c|c|c|c|}
\hline & Baseline & HDBR + 89 & Recovery day +5 \\
\hline RR interval (ms) & $942 \pm 27$ & $842 \pm 20^{*}$ & $869 \pm 25^{*}$ \\
\hline $\mathrm{SAP}(\mathrm{mmHg})$ & $123.7 \pm 3.7$ & $114.4 \pm 2.9$ & $118.1 \pm 2.6$ \\
\hline MSNA (burst/min) & $40.9 \pm 3.8$ & ND & $28.8 \pm 7.4^{*}$ \\
\hline MSNA (burst/60 beats) & $39.1 \pm 4.0$ & ND & $24.2 \pm 5.8^{*}$ \\
\hline \multicolumn{4}{|l|}{$\mathrm{R}-\mathrm{R}$ variability } \\
\hline Total power $\left(\mathrm{ms}^{2}\right)$ & $3466 \pm 455$ & $2239 \pm 386^{*}$ & $3357 \pm 444$ \\
\hline LF (normalized units) & $61.4 \pm 3.5$ & $62.9 \pm 4.8$ & $62.1 \pm 4.6$ \\
\hline HF (normalized units) & $34.4 \pm 3.7$ & $29.5 \pm 4.9$ & $32.8 \pm 4.1$ \\
\hline \multicolumn{4}{|l|}{ SAP variability } \\
\hline Variance $\left(\mathrm{mmHg}^{2}\right)$ & $37 \pm 9$ & $23 \pm 6$ & $34 \pm 6$ \\
\hline $\mathrm{LF}\left(\mathrm{mmHg}^{2}\right)$ & $3.2 \pm 0.6$ & $5.1 \pm 1.4$ & $3.6 \pm 0.7$ \\
\hline $\mathrm{HF}\left(\mathrm{mmHg}^{2}\right)$ & $2.1 \pm 0.5$ & $1.1 \pm 0.3$ & $1.1 \pm 0.2$ \\
\hline RR-SAP LF $K^{2}$ & $0.72 \pm 0.06$ & $0.68 \pm 0.05$ & $0.87 \pm 0.03$ \\
\hline RR-SAP HF $K^{2}$ & $0.83 \pm 0.03$ & $0.77 \pm 0.06$ & $0.88 \pm 0.03$ \\
\hline $\mathrm{BRS}(\mathrm{ms} / \mathrm{mmHg})$ & $25.3 \pm 3.8$ & $13.8 \pm 1.3^{*}$ & $23.8 \pm 2.1$ \\
\hline \multicolumn{4}{|l|}{ MSNA variability } \\
\hline Variance $\left(\mathrm{AU}^{2}\right)$ & $0.31 \pm 0.13$ & ND & $0.16 \pm 0.03$ \\
\hline LF (normalized units) & $33.7 \pm 1.7$ & ND & $30.6 \pm 4.6$ \\
\hline HF (normalized units) & $39.6 \pm 3.4$ & ND & $35.2 \pm 6.0$ \\
\hline MSNA-RR LF $K^{2}$ & $0.59 \pm 0.07$ & ND & $0.69 \pm 0.06$ \\
\hline MSNA-RR HF $K^{2}$ & $0.44 \pm 0.07$ & ND & $0.48 \pm 0.05$ \\
\hline MSNA-SAP LF $K^{2}$ & $0.62 \pm 0.09$ & ND & $0.68 \pm 0.06$ \\
\hline MSNA-SAP HF $K^{2}$ & $0.49 \pm 0.08$ & ND & $0.44 \pm 0.05$ \\
\hline
\end{tabular}

Data are given as mean $\pm \mathrm{SE}$. $\mathrm{AU}$, arbitrary units; BRS, baroreceptor reflex sensitivity; HDBR, head down bed rest; HF, high-frequency component; LF, low-frequency component; MSNA, muscle sympathetic nerve activity; ND, not done; SAP, systolic arterial pressure. ${ }^{*} P<0.05$ versus baseline.

heart rate and was also maintained after normalizing for burst area $(P<0.001)$, in order to account for unknowns, such as variations in electrode-nerve contact. At variance with our study, other reports showed no significant changes [12] or even an increase [16] in resting MSNA after bed rest. Part of these inconsistencies might be related to the different durations of HDBR, different time points of measurements and to different assessment techniques, MSNA and its variability in the present study being assessed automatically by an ad-hoc software tool [33]. Anyway, the finding of a reduction in norepinephrine turnover reported after HDBR [15] together with a reduced sympathetic activity in brainstem regions involved in cardiovascular control in rats after prolonged head-down suspension [34], suggesting a possible central inhibition of efferent sympathetic activity, would support our findings of a reduced peripheral sympathetic drive after prolonged HDBR and inactivity. On the contrary, the consistent observation that oscillatory profile of RR and MSNA variability (as assessed by the power of spectral components, see Figs 2 and 5) does not change over time, combined with the strong coherence between couples of hemodynamic variables among themselves (RR and SAP) and with MSNA [25] (Table 1), suggests that, at least within the constraints of the explored window, resting central cardiovascular oscillatory regulation is not affected by prolonged HDBR. The analysis of only RR variability could explain the discrepant conclusions reached by others [32].
The extent to which our findings on tonic ANS regulation, particularly that of a decrease in sympathetic efferent activity, are due to inactivity, that is, bed-rest confinement, or to the associated head-down position, that is, altered gravitational pull on the blood column, cannot be precisely teased out. The possibility that changes in blood volume and increase in venous pooling, which normally occur with HDBR, could have affected MSNA results through alterations in cardiopulmonary baroreceptors activity, which normally exerts a tonic restraint on peripheral sympathetic activity, could not be ruled out. However, the hypovolemia occurring with prolonged HDBR should unload volume receptors in the cardiopulmonary region, which should result in an increase in MSNA [16], rather than a decrease, as observed in the present investigation. Again, the reduction in MSNA after HDBR could have been due to an attenuated arterial baroreflex control of peripheral sympathetic activity. Although we did not measure baroreflex control of MSNA as a part of the study, a prior report indicated that 120 days of HDBR resulted in an augmentation in baroreflex control of MSNA [16]. The maintained baroreflex control of heart rate at REC5 also suggests a functional preservation of arterial baroreflexes few days after HDBR in our participants. Several studies have indicated that the effects of head-down tilt are qualitatively similar to those of horizontal bed rest, though the adaptation is accelerated in the former, particularly during short-term exposure [9]. In addition, some effects of head down, such as reduced total blood volume, may vanish after some days of readaptation to the gravitational field [9].

In brief, HDBR inactivity and fluid shifts have important influences on autonomic cardiovascular regulation, though our study cannot disentangle in detail the specific role of either factor.

\section{Effects on standardized stimuli Responses to handgrip}

The pressor and heart rate responses to handgrip were in this study limited, by design, because of the light level (25\% of maximal voluntary contraction) that was allowed by the overall experimental plan. In spite of this limitation, it was clear that the pressor increase was reduced (Fig. 2) at $\mathrm{REC}+5(1.6 \pm 1.1 \mathrm{mmHg})$ as compared with BDC $(14.4 \pm 3.1 \mathrm{mmHg}, P<0.05)$, supporting further the notion of an inhibitory influence of HDBR on vasoconstriction. In addition, the MSNA response showed a minimum during $\mathrm{REC}+5$, suggesting that the previous observations [14] of a reduced pressor responses obtained with a shorter HDBR exposure might be extended to tonic MSNA response during longer HDBR conditions. Clearly, our findings relate to small masses' light static exercise and should not be generalized to other kinds of exercise, eventually involving larger muscle masses or greater exercise intensity $[35,36]$. 
Fig. 2

\section{Rest and Handgrip}
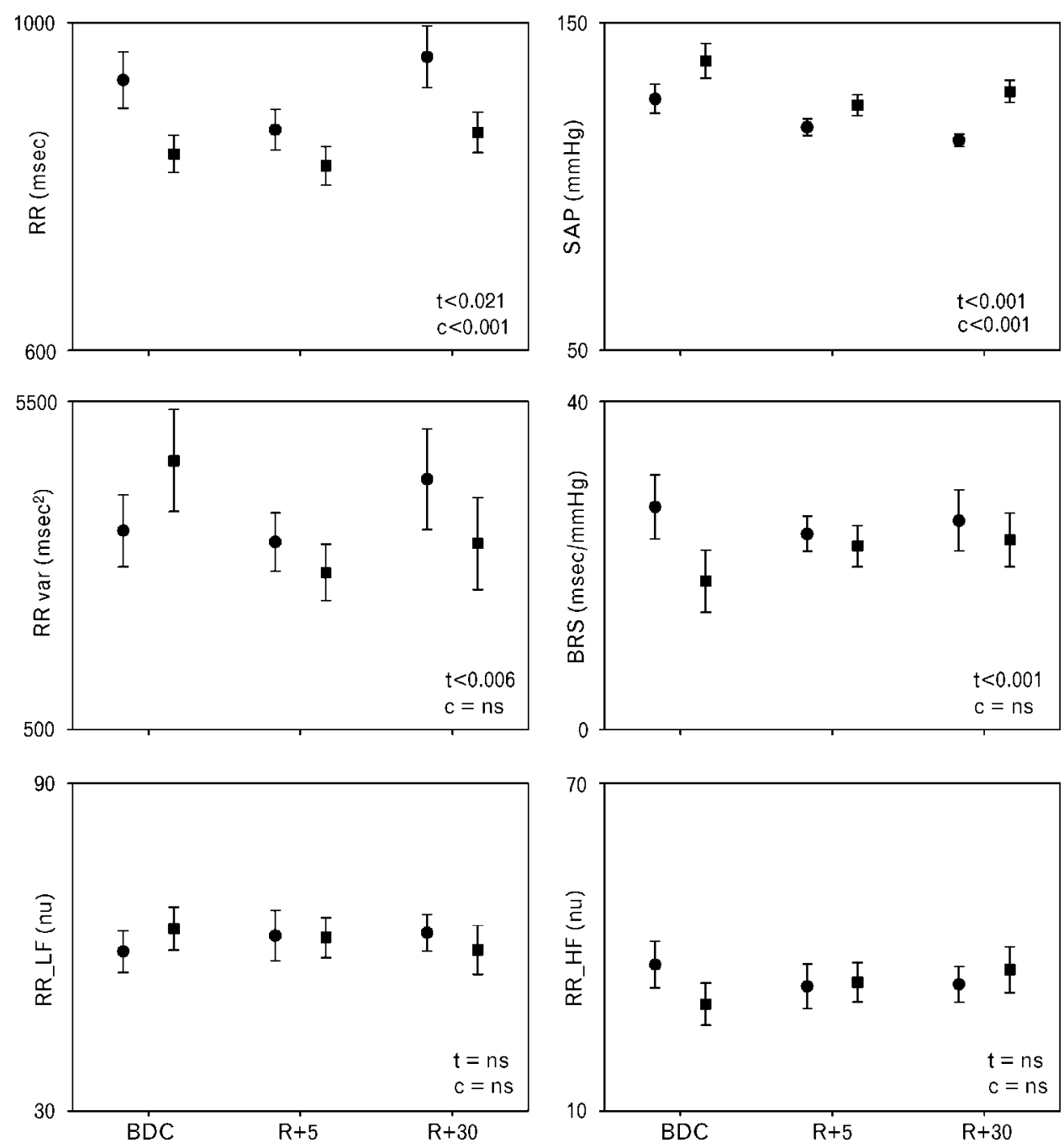

Effects of prolonged inactivity and head down bed rest on RR interval and indices of variability (RR var, RR_LF and RR_HF) as well as on systolic arterial pressure and baroreceptor reflex sensitivity. Data are means $\pm S E M$, and values are plotted at baseline data collection and early ( $R+5)$ and late $(R+30)$ recovery after the end of exposure at HDBR. Both values obtained at baseline (O) and during submaximal handgrip ( $\square$ ) are presented. Significance of changes, as a function of time and of condition, that is, rest against handgrip, is indicated. Significant interaction was present for RR interval and SAP. BRS, baroreceptor reflex sensitivity; HF, high frequency; LF, low frequency; ns, nonsignificant; RR; SAP, systolic arterial pressure; var, variance.

\section{Responses to active standing and lower body negative pressure}

We did not observe symptoms of orthostatic intolerance or marked decreases in blood pressure during standing, as it might occur after prolonged bed confinement. A well preserved orthostatic tolerance might be related to the relative short period of standing required by our protocol: had we kept our participants upright for a longer duration or had we exposed them to this stimulus at an earlier time (e.g., day 0 of REC), some cases of orthostatic intolerance might have occurred. In addition, the clinical selection procedure at MEDES might have avoided including participants known to be prone to fainting, thus avoiding the inclusion of participants susceptible to orthostatic intolerance, a condition often reported after HDBR. Nevertheless, it is remarkable that the oscillatory cardiovascular responses to orthostatic challenges were well preserved, as it occurred for the MSNA oscillations during handgrip.

Recent studies from Hamburg et al. [22], however, showed that bed rest, with the inherent inactivity, induced a clear 
Fig. 3

Rest and Handgrip
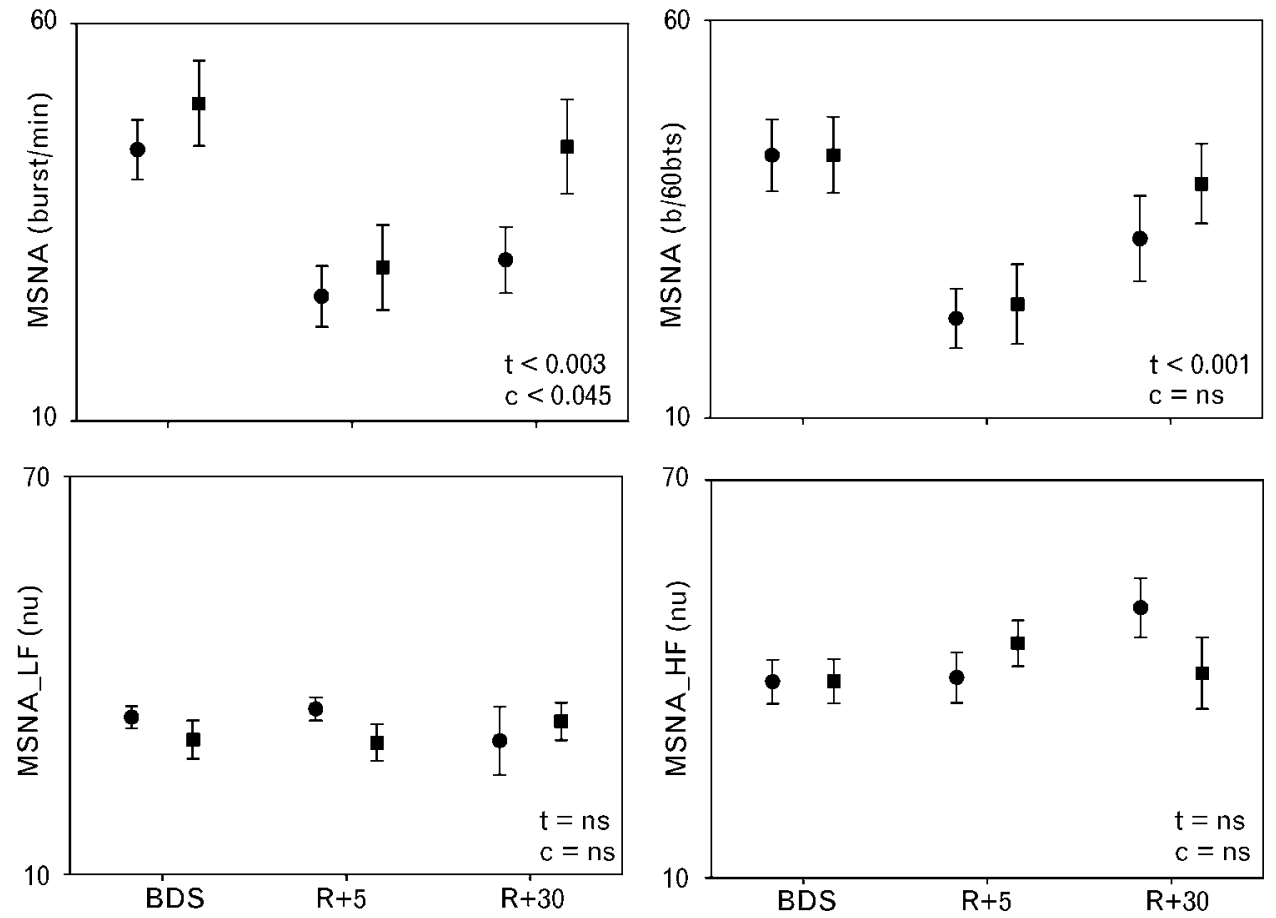

Effects of prolonged inactivity and head down bed rest on muscle sympathetic nerve activity and indices of variability (MSNA_LF and MSNA_HF). Data are means \pm SEM, and values are plotted at BDC (baseline data collection) and early $(R+5)$ and late $(R+30)$ recovery after the end of exposure at HDBR. Both values obtained at baseline (O) and during sub maximal handgrip ( $\square$ ) are presented. HF, high frequency; LF, low frequency; MSNA, muscle sympathetic nerve activity. Significance of changes as a function of time and of condition, that is, rest against handgrip, is indicated. Significant interaction was present for MSNA (burst/min) and MSNA (burst/60 beats).

state of insulin resistance. A state of insulin resistance has been reported in association with a selective impairment in BRS in patients with metabolic syndrome [20] and in normoglicemic offspring of diabetic patients [37] without concomitant alterations in the oscillatory cardiac profile. The same has been observed in inactive hypertensive children [19]. Thus, we could speculate that at least the selective reduction of baroreflex gain observed during and after exposure to HDBR and inactivity might be linked to a subclinical dysmetabolic state.

The similarity of responses to LBNP and to active standing observed before and after HDBR merits a comment. We compared active standing with the LBNP stimulation of $-60 \mathrm{mmHg}$, the maximal-tolerated negative pressure. We observed that active standing induced more marked effects on RR interval, BRS and on oscillatory indices, consistently over time (Fig. 3). The notable exception was a smaller starting RR interval at REC day +5 . Accordingly, the frequent use of LBNP as a model of an orthostatic challenge might be regarded as suboptimal, at least from an autonomic perspective.

\section{Limitations of the study}

Some measures (MSNA) or stimuli (LBNP and standing) were performed only before and after HDBR confinement. As previously mentioned, in our study, the head-down position added to the effects of pure inactivity. The headdown position could have affected volume receptors of the heart, and this might have influenced our results. However, this would not alter our main finding that prolonged HDBR and attendant inactivity impair tonic cardiovascular regulation, while sparing oscillatory rhythms. As an example, SAP and MSNA during handgrip showed a minimum response at early REC after HDBR, when participants resumed the supine posture, whereas their oscillatory components were still unaffected.

In conclusion, we investigated simultaneously peripheral sympathetic nerve activity and cardiovascular variables along with their oscillatory rhythms in response to prolonged inactivity (as induced by HDBR). Our data imply that prolonged inactivity and gravity pull of HDBR induce short-lasting changes in peripheral and cardiac average neural activity leaving unaffected the central rhythms 
Rest, LBNP and Stand
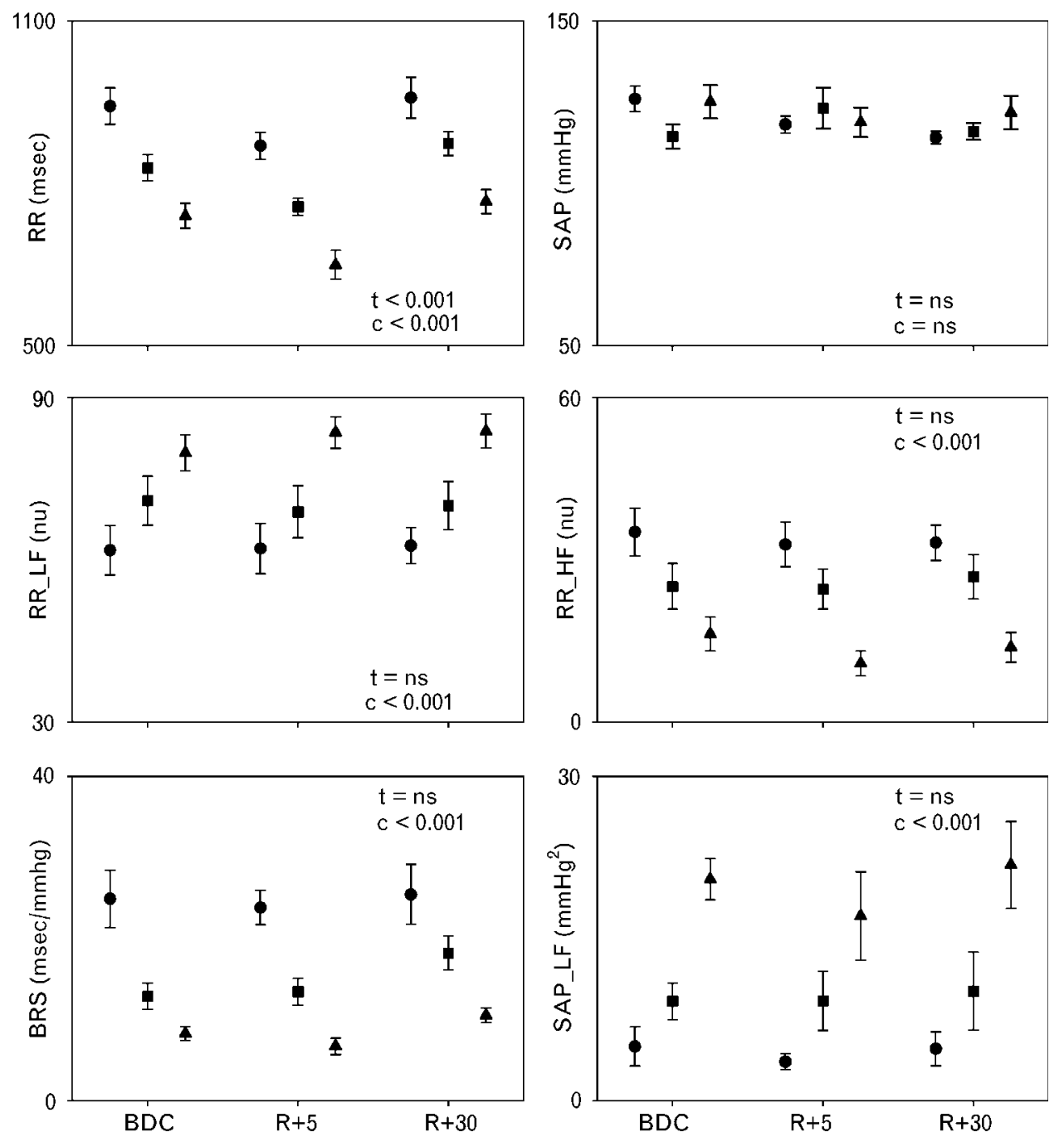

Effects of prolonged inactivity and head down bed rest on RR interval and indices of variability (RR_LF and RR_HF) as well as on systolic arterial pressure and systolic arterial pressure variability (SAP_LF) and baroreceptor reflex sensitivity. Data are means \pm SEM, and values are plotted at before data collection (BDC) and early $(R+5)$ and late $(R+30)$ recovery after the end of exposure at HDBR. Both values obtained at baseline $(O)$, during LBNP $(\boldsymbol{\square})$ and during standing $(\boldsymbol{\Lambda})$ are presented. Significance of changes, as a function of time and of condition, such as rest, LBNP and standing, is indicated. Significant interaction was present for RR interval, RR_LF, RR_HF, BRS and SAP_LF. BRS, baroreceptor reflex sensitivity; HF, high frequency; LBNP, lower body negative pressure; LF, low frequency; ns, nonsignificant; SAP, systolic arterial pressure; var, variance.

governing cardiovascular oscillations and their responses, a critical sign of a maintained autonomic competence.

\section{Perspectives}

The epidemic of inactivity is rapidly expanding in the global world, contributing to the growing toll of cardiovascular diseases, through an increase of prevalence of obesity, diabetes and atherosclerosis and their consequences [38], also in the low and middle income countries [39]. Among the new behavioral risk factors (e.g., inactivity, stress and eating habits), few studies addressed specifically the effects of inactivity, particu- larly from the functional side [40]. The findings of the present investigation that rhythmic autonomic profiles are not altered by inactivity, as produced by prolonged HDBR, while tonic levels of autonomic activity are affected, possibly by way of a dysmetabolic pathway, might prompt more focused long-term studies on the effects of specific countermeasures. If lifestyle interventions such as exercise, or specific drugs, might provide the best approach to personalize treatment [41], particularly in the field of hypertension, where even high normal levels increase overall risk [42], remains to be elucidated. 
Fig. 5

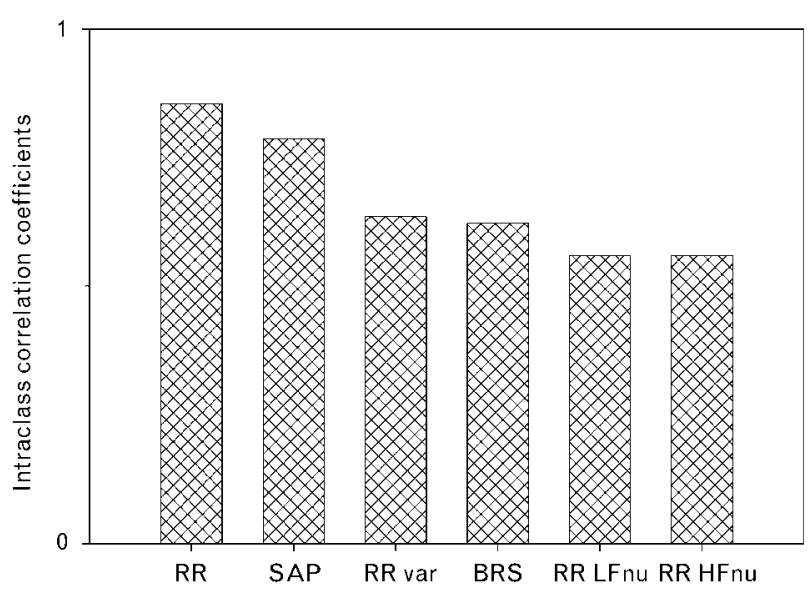

Intraclass correlation coefficients for RR, RR variability, arterial pressure and baroreceptor reflex sensitivity, considering the effects of time. Intraclass correlation coefficients were computed at rest, across baseline data collection (BDC), head down bed rest (HDBR +5 , $\mathrm{HDBR}+45, \mathrm{HDBR}+89)$ and recovery $(R+5, \mathrm{R}+30)$. BRS, baroreceptor reflex sensitivity; HDBR, head down bed rest; HF, high frequency; LF, low frequency; SAP, systolic arterial pressure; var, variance.

\section{Acknowledgements}

The valuable contribution of Dr Sara Bonizzato is gratefully recognized.

The study was partially supported by Agenzia Spaziale Italiana (ASI) (Project on Disorders of Motor and Cardiorespiratory Control (DCMC).

There was no conflict of interests.

\section{References}

1 Booth FW, Lees FW, Simon J. Fundamental questions about genes, inactivity, and chronic diseases. Physiol Genomics 2007; 28:146-157.

2 Booth FW, Gordon SE, Carlson CJ, Hamilton MT. Waging war on modern chronic diseases: primary prevention through exercise biology. J Appl Physiol 2000; 88:774-787.

3 Haskell WL, Lee IM, Pate RR, Powell KE, Blair SN, Franklin BA, et al. Physical activity and public health: updated recommendation for adults from the American College of Sports Medicine and the American Heart Association. Circulation 2007; 116:1081-1093.

4 Warburton DER, Nicol CW, Bredin SSD. Health benefits of physical activity: the evidence. CMAJ 2006; 174:801-809.

5 Pagani M, Somers VK, Furlan R, Dell'Orto S, Conway J, Baselli G, et al. Changes in autonomic regulation induced by physical training in mild hypertension. Hypertension 1988; 12:600-610.

6 Carter JB, Banister EW, Blaber AP. The effect of age and gender on heart rate variability after endurance training. Med Sci Sports Exerc 2003; 35:1333-1340

7 Hjalmarson $\AA$. Heart rate: an independent risk factor in cardiovascular disease. Eur Heart J (Suppl) 2007; 9:F3-F7.

8 Sandler H, Vernikos J, editors. Inactivity: physiological effects. New York: Academic Press; 1986. p. 205.

9 Blomquist CG, Stone HL. Cardiovascular adjustments to gravitational stress. In: Sheperd JT, Abboud FM, editors. Handbook of physiology. Section 2: The cardiovascular system (vol. III). Bethesda, Maryland: American Physiological Society; 1983. pp. 1025-1063.

10 Ferretti G, Antonutto G, Denis C, Hoppeler H, Minetti AE, Narici MV Desplaches $D$. The interplay of central and peripheral factors in limiting maximal 02 consumption in man after prolonged bed rest. Meds Sci Sport Exerc 1997; 29:207-215.
11 Pavy-Le Traon A, Herr M, Narici MV, Rittweger J, Verkinos J. From space to Earth: advances in human physiology from 20 years of bed rest studies (1986-2006). Eur J Appl Physiol 2007; 101:143-194.

12 Crandall CG, Engelke KA, Convertino VA, Raven PB. Aortic baroreflex control of heart rate after 15 days of simulated microgravity exposure. J Appl Physiol 1994; 77:2134-2139.

13 Kamiya A, Michikami D, Shiozawa T, Iwase S, Hayano J, Kavada T, et al. Bed rest attenuates sympathetic and pressor responses to isometric exercise in antigravity leg muscles in humans. Am J Physiol Regul Integr Comp Physiol 2004; 286:R844-R850.

14 Pagani M, lellamo F, Lucini D, Cerchiello M, Castrucci F, Pizzinelli P, et al. Selective impairment of exitatory pressor responses after prolonged simulated microgravity in humans. Auton Neurosci 2001; 91:85-95.

15 Goldstein DS, Vernikos J, Holmes C, Convertino VA. Catecholaminergic effects of prolonged head-down bed rest. J Appl Physiol 1995; 78:10231029.

16 Kamiya A, Iwase S, Kitazawa H, Mano T, Vinogradova OL, Kharchenko IB. Baroreflex control of muscle sympathetic nerve activity after 120 days of 6 degrees head-down bed rest. Am J Physiol Regul Integr Comp Physiol 2000; 278:R445-R452.

17 Hughson RL, Yamamoto Y, Maillet A, Fortrat JO, Pavy-Le Traon A, Butler C et al. Altered autonomic regulation of cardiac function during head-up till after 28-day head-down bed-rest with counter-measures. Clin Physiol 1994; 14:201-204.

18 Van de Borne P, Montano N, Narkiewicz K, Degaute JP, Pagani M, Somers VK. Sympathetic rythmicity in cardiac transplant recipient. Circulation 1999; 99:1606-1610.

19 Genovesi S, Pieruzzi F, Giussani M, Tono V, Stella A, Porta A, et al. Analysis of heart period and arterial pressure variability in childhood hypertension: evidence for a key role of baroreflex impairment. Hypertension 2008; 51:1289-1294.

20 Lucini D, Cusumano G, Bellia A, Kozakova M, Di Fede G, Lauro R, Pagani $M$. Is reduced baroreflex gain a component of the metabolic syndrome? Insights from the Linosa study. J Hypertens 2006; 3424:361 370.

21 Pagani M, Malliani A. Interpreting oscillations of muscle sympathetic nerve activity and of heart rate variability. J Hypertens 2000; 18:17091719.

22 Hamburg NM, Mc Mackin CJ, Huang AL, Shenouda SM, Widlansky ME, Schulz $\mathrm{E}$, et al. Physical inactivity rapidly induces insuline resistance and microvascular dysfunction in healthy volunteers. Arterioscler Thromb Vasc Biol 2007; 27:2650-2656.

23 Pagani M, Lombardi F, Guzzetti S, Rimoldi O, Furlan R, Pizzinelli P, et al. Power spectral analysis of heart rate and arterial pressure variabilities as a marker of sympathovagal interaction in man and conscious dog. Circ Res 1986; 58:178-193.

24 Bertinieri G, Di Rienzo M, Cavallazzi A, Ferrari A, Pedotti A, Mancia G. Evaluation of baroreceptor reflex by blood pressure monitoring in unanesthetized cats. Am J Physiol 1988; 254:H377-H383.

25 Pagani M, Montano N, Porta A, Malliani A, Abboud FM, Birkett CL, Somers VK. Relationship between spectral components of cardiovascular variabilities and direct measures of muscle sympathetic nerve activity in humans. Circulation 1997; 95:1441-1448.

26 Porta A, Guzzetti S, Montano N, Pagani M, Somers VK, Malliani A, et al. Information domain analysis of cardiovascular variability signals: evaluation of regularity, synchronisation and co-ordination. Med Biol Eng Comput 2000; 38:180-188.

27 Van de Borne P, Montano N, Zimmerman BG, Pagani M, Somers VK. Relationship between repeated measures of hemodynamics, muscle sympathetic nerve activity and the spectral oscillations. Circulation 1997; 96:4326-4332.

28 Task Force of the European Society of Cardiology and the North American Society of Pacing and Electrophysiology. Heart rate variability. Standards of measurement, physiological interpretation, and clinical use. Circulation 1996; 93:1043-1065.

29 Malliani A, Pagani M, Lombardi F, Cerutti S. Cardiovascular neural regulation explored in the frequency domain. Circulation $1991 ; \mathbf{8 4}: 482-$ 492.

30 Iwasaki K-I, Zhang R, Zuckerman JH, Pawelczyk JA, Levine BD. Effect of head-downtilt bed rest and hypovolemia on dynamic regulation of heart rate and blood pressure. Am J Physiol Regul Integrative Comp Physiol 2000; 279:R2189-R2199.

31 Migeotte P-F, Prisk GK, Paiva M. Microgravity alters respiratory sinus arrhythmia and short-term heart rate variability in humans. Am J Physiol Heart Circ Physiol 2003; 284:H1995-H2006.

32 Shoemaker JK, Hogeman CS, Leuenberger U, Herr MD, Gray K, Silber DH, Sinoway L. Sympathetic discharge and vascular resistance after bed rest. J Appl Physiol 1998; 84:612-617. 
33 Badilini F, Pagani M, Porta A. Heartscope: a software tool addressing autonomic nervous system regulation. Comput Cardiol 2005; 32:259262.

34 Fagette S, Somody L, Bouzeghrane F, Cottet-Emard J, Gharib C,

Gauquelin G. Central and peripheral sympathetic activities in rats during recovery from simulated weightlessness. J Appl Physiol 1995; 79:19911997.

35 lellamo F. Neural mechanisms of cardiovascular regulation during exercise. Auton Neurosci 2001; 90:66-75.

36 lellamo F, Di Rienzo M, Lucini D, Legramante JM, Pizzinelli P, Castiglioni P, et al. Muscle metaboreflex contribution to cardiovascular regulation during dynamic exercise in microgravity: insights from mission STS-107 of the space shuttle Columbia. J Physiol 2006; 572:829-838.

37 lellamo F, Tesauro M, Rizza S, Aquilani S, Cardillo C, lantorno M, et al. Concomitant impairment in endothelial function and neural cardiovascular regulation in offspring of type 2 diabetic subjects. Hypertension 2006; 48:418-423.

38 Haffner S, Taegtmeyer H. Epidemic obesity and the metabolic syndrome. Circulation 2008; 108:1541-1545.

39 Abegunde DO, Mathers DC, Adam T, Ortegon M, Strong K. The burden and costs of chronic diseases in low-income and middle-income countries. Lancet 2007; 370:1929-1938.

40 Hamilton MT, Hamilton DG, Zderic TW. Exercise physiology versus inactivity physiology: an essential concept for understanding lipoprotein lipase regulation. Exerc Sport Sci Rev 2004; 32:161-166.

41 Turner ST, Schwartz GL, Boerwinkle E. Personalized medicine for high blood pressure. Hypertension 2007; 50:1-5.

42 Vasan RS, Larson MG, Leip EP, Evans JC, O'Donnel CJ, Kannel WB, Levy D. Impact of high normal blood pressure on the risk of cardiovascular disease. N Engl J Med 2001; 345:1291-1297. 